\title{
STABILITY PROPERTIES FOR GENERALIZED FRACTIONAL DIFFERENTIAL SYSTEMS
}

\author{
DENIS MATIGNON
}

\begin{abstract}
In the last decades, fractional differential equations have become popular among scientists in order to model various stable physical phenomena with anomalous decay, say that are not of exponential type. Moreover in discrete-time series analysis, so-called fractional ARMA models have been proposed in the literature in order to model stochastic processes, the autocorrelation of which also exhibits an anomalous decay. Both types of models stem from a common property of complex variable functions: namely, multivalued functions and their behaviour in the neighborhood of the branching point, and asymptotic expansions performed along the cut between branching points. This more abstract point of view proves very much useful in order to extend these models by changing the location of the classical branching points (the origin of the complex plane, for continuous-time systems). Hence, stability properties of and modelling issues by generalized fractional differential systems will be adressed in the present paper: systems will be considered both in the time-domain and in the frequency-domain; when necessary a distinction will be made between fractional differential systems of commensurate and incommensurate orders.
\end{abstract}

RÉsumÉ. Ces dernières années, les équations différentielles fractionnaires ont été de plus en plus utilisées par les scientifiques désireux de modéliser divers phénomènes physiques stables mais présentant une décroissance lente, c'està-dire qui ne soit pas de type exponentiel. D'autre part, dans le domaine de l'analyse des séries temporelles, des modèles ARMA fractionnaires ont été proposés de façon à modéliser des processus stochastiques dont l'autocorrélation est aussi à décroissance lente. Ces deux types de modèles proviennent d'une propriété commune des fonctions de la variable complexe : à savoir, les fonctions multivaluées et leur comportement au voisinage du point de branchement, ainsi que des développements asymptotiques effectués le long de la coupure qui relie les points de branchement. Ce point de vue plus abstrait révèle toute son utilité lorsqu'on veut étendre ces modèles en changeant la position des points de branchement classiques (l'origine du plan complexe, pour les systèmes en temps continu). Ainsi, nous étudierons les propriétés de stabilité des systèmes différentiels fractionnaires généralisés et les conséquences sur la modélisation : nous considérerons les systèmes tant dans le domaine temporel que dans le domaine fréquentiel ; et si nécessaire nous ferons la distinction entre systèmes différentiels fractionnaires d'ordres commensurables ou incommensurables.

\section{INTRODUCTION}

\subsection{Context and Motivation}

In the fields of continuous-time modelling, fractional derivatives have proved useful in linear viscoelasticity, acoustics, rheology, polymeric chemistry (see e.g. [24])... For a treatment of so-called fractional differential equations (FDEs), we refer to [27, chap. 8, sec. 42], [19, chap. 5 \& 6] and [10, 12].

\footnotetext{
(C) Société de Mathématiques Appliquées et Industrielles. Typeset by $\mathrm{HT}_{\mathrm{E}} \mathrm{X}$.

D. Matignon: ENST, Dept. TSI \& CNRS, URA 820. 46, rue Barrault, 75634 Paris Cedex 13, France. Email: matignon@tsi.enst.fr. URL: http://н日н-sig.enst.fr/ matignon.
} 
There has been some recent advances in control theory of such systems (see e.g. [12] for stability questions, [16] for controllability and observability considerations and [17] for observer-based controller design), together with interesting applications (see $[18,22])$.

Turning to the infinite dimension (i.e. dealing with FPDEs and introducing a so-called fractional modal decomposition) has been motivated by the example of a wave equation in viscothermal medium (see $[10,15,11,14]$ ).

Moreover, an interesting idea of generalized fractional differential systems appeared in [30] in a stochastic framework; in this approach however, new branching points are definitely singular points like poles for meromorphic functions (in the sense that the modulus of the complex function goes to infinity in the vicinity of the branching point).

\subsection{TECHNiQues}

It is the very multivalued nature of the transfer function that gives its richness to the model; thus, moving the branching point and studying the consequences both from spectral and time-domain point of views proves useful when one is interested in extending these models. The techniques involved in this work are of analytic nature: we use mostly distributions theory in the sense of $\mathrm{Schwartz}^{1}$ (see e.g. [28, chap. II \& III], [8, chap. 1, sec. $3.2 \& 5.5]$ ) for the continuous-time domain, complex variable theory (see [3]), asymptotic expansions (see [4, chap. II]) and special functions (see e.g. $[20,1,7])$. The very nature of fractional integrals and derivatives can be better understood in the context of so-called diffusive realisations, which will also be used in the sequel (see e.g. [23, 22, 9, 21]).

\subsection{Problems at stake}

Different problems will be considered in the present paper. We will focus on stability properties of fractional differential systems; only external stability will be considered.

For systems of commensurate order of derivatives, an algebraic approach combined with the use of asymptotic results (carefully proved or only found in the literature) gives a well-known stability result (see [10, Appendix B]).

The problem of stability then seems to be left wide open in the case of fractional differential systems of incommensurate orders of derivatives and also in the case of generalized fractional differential systems. From a careful asymptotic analysis, we are able to give a general result of stability and also to provide a constructive method to determine the asymptotic expansion of the impulse response of the system under study. Moreover some concrete examples of first and second order systems with damping of fractional order are carefully examined, using techniques of analytic nature, such as energy methods and diffusive realisations.

\subsection{Outline}

The paper is organized as follows: in section 2, we study fractional differential systems in continuous time; we start with some basic definitions and properties in subsection 2.1, we then address the problem of stability for systems of commensurate order in subsection 2.2; more general stability results, i.e. for systems of incommensurate orders are to be found in subsection 2.3 , they give rise to some case studies in subsection 2.4, where it is shown that algebraic and analytic approaches can be combined in a very creative way.

\footnotetext{
${ }^{1}$ an attempt to use Mikusinski calculus has been presented in [6]; for a translation between distributions theories, see [5, p. 16 \& 62 especially] and [2]. 
Then, in section 3, generalized fractional differential systems are introduced, which amounts to moving the location of the classical branching point $s=0$; generalized basic elements are first examined in subsection 3.1, and they are combined in a natural way to build up generalized fractional differential systems in subsection 3.2; finally in subsection 3.3, other generalized fractional differential systems are introduced, namely when more than one branching point is being used; some examples of application to Bessel functions are also investigated.

\section{Fractional differential systems in continuous time}

\subsection{Definitions}

For more details, the reader can refer to $[10$, Appendix A], [8, chapter 1 , section 5.5$]$ and $[27$, chapter 2 , section 8$]$.

\subsubsection{Fractional integrals.}

Definition 2.1. We define $Y_{\alpha}$, the convolution kernel of order $\alpha$ for fractional integrals:

$$
\text { for } \quad \alpha>0, \quad Y_{\alpha}(t) \triangleq \frac{t_{+}^{\alpha-1}}{\Gamma(\alpha)} \in L_{\mathrm{loc}}^{1}\left(\mathbb{R}^{+}\right)
$$

where $\Gamma$ is the well-known Euler Gamma function.

With this notation, the fractional integral of order $\alpha$ of a causal function or causal distribution $f$ is:

$$
I^{\alpha} f \triangleq Y_{\alpha} \star f
$$

Remark 2.2. This definition coincides with the so-called Riemann-Liouville integral of fractional order $\alpha$.

Proposition 2.3. The Laplace transform of $Y_{\alpha}$ is:

$$
\mathcal{L}\left[Y_{\alpha}\right](s)=s^{-\alpha} \quad \text { for } \Re e(s)>0
$$

We have the important convolution property $Y_{\alpha} \star Y_{\beta}=Y_{\alpha+\beta}$ for $\alpha>0$ and $\beta>0$, which translates into a sequentiality property: $I^{\alpha} \circ I^{\beta}=I^{\alpha+\beta}$.

Proof. straightforward.

REMARK 2.4. In (2.3), the function $s \mapsto s^{\alpha}$ of the complex variable $s$ is defined with no ambiguity as the analytic continuation of $x \mapsto x^{\alpha}$ on $\mathbb{R}^{+}$into the strip of convergence of the Laplace transform, namely $\Re e(s)>0$. Hence, for $s=\rho \exp (i \theta)$ and $|\theta|<\pi / 2, s^{\alpha}$ has the analytic value $s^{\alpha}=\rho^{\alpha} \exp (i \alpha \theta)$.

\subsubsection{Fractional derivatives in the Sense of Distributions.}

Definition 2.5. We define $Y_{-\alpha}$, the causal distribution - or generalized function in the sense of Schwartz (see [28, chapters II \& III] and [8, chapter 1, section 3.2]) as the unique convolutive inverse of $Y_{+\alpha}$ in the convolution algebra $\mathcal{D}_{+}^{\prime}(\mathbb{R})$; with the use of $\delta$ - the Dirac distribution - which is the neutral element of convolution, this reads:

$$
Y_{+\alpha} \star Y_{-\alpha}=\delta
$$

The causal distribution $Y_{-\alpha}$ is in fact the finite part of a divergent integral (in the sense of Hadamard); more precisely:

$$
Y_{-\alpha} \triangleq \mathrm{fp}\left\{\frac{t_{+}^{-\alpha-1}}{\Gamma(-\alpha)}\right\}
$$


With this notation, the fractional derivative of order $\alpha$ of a causal function or distribution $f$ is:

$$
D^{\alpha} f \triangleq Y_{-\alpha} \star f
$$

REMARK 2.6. This definition does not always coincide with the so-called RiemannLiouville derivative of fractional order $\alpha$; in fact it depends on the regularity of the function $f$ at the origin. In particular with Riemann-Liouville derivatives, the fundamental sequentiality property can be violated, moreover it is only a leftinverse of the fractional integral. A smooth fractional derivative (sometimes called the Caputo derivative) can also be introduced for regularity requirements when taking initial conditions into account. For a comparison between these notions, see e.g. [12].

REMARK 2.7. Only in the integer case $\alpha=n \in \mathbb{N}$ do we get distributions, the support of which is localized at 0 ; otherwise, the support is $\mathbb{R}^{+}$. We have:

$$
Y_{-n}=\delta^{(n)}
$$

Proposition 2.8. The Laplace transform of $Y_{-\alpha}$ is:

$$
\mathcal{L}\left[Y_{-\alpha}\right](s)=s^{\alpha} \quad \text { for } \quad \Re e(s)>0
$$

We then have the important convolution property $Y_{\alpha} \star Y_{\beta}=Y_{\alpha+\beta}$ for any real numbers $\alpha, \beta$, which translates into a sequentiality property: $D^{\alpha} \circ D^{\beta}=D^{\alpha+\beta}$.

Proof. straightforward.

REMARK 2.9. The latter property ensures that derivation in the sense of causal distributions is sequential in the sense of [19, section VI.4], thus allowing for a fully algebraic treatment of FDEs of commensurate order (see theorem 2.21 in $\$ 2.2 .3$ ).

2.1.3. Fundamental solutions of the FraCtional DifFERENTIAL operator. In the sequel, $0<\alpha<1$ will be assumed. Let us now define the eigenfunctions of the previous operator $D^{\alpha}$ (see [10, Appendix B] and [26, 20, 1] for more details and an extensive study of these special functions together with existing links with generalized Mittag-Leffler functions and their derivatives).

DeFinition 2.10. We define $\mathcal{E}_{\alpha}(\lambda, t)$ as the fundamental solution of the operator $D^{\alpha}-\lambda$ :

$$
\mathcal{E}_{\alpha}(\lambda, t) \triangleq \sum_{k=0}^{\infty} \lambda^{k} Y_{(k+1) \alpha}=t_{+}^{\alpha-1} \sum_{k=0}^{\infty} \frac{\left(\lambda t_{+}^{\alpha}\right)^{k}}{\Gamma((k+1) \alpha)}
$$

the Laplace transform of which is $\left(s^{\alpha}-\lambda\right)^{-1}$ for $\Re e(s)>a_{\lambda}$.

It follows that:

$$
D^{\alpha} \mathcal{E}_{\alpha}(\lambda, t)=\lambda \mathcal{E}_{\alpha}(\lambda, t)+\delta
$$

But in the case of multiple root, we need to define the $j$-th convolution of $\mathcal{E}_{\alpha}(\lambda, t)$, namely:

Definition 2.11. For integer $j \geq 1$, we define $\mathcal{E}_{\alpha}^{\star j}(\lambda, t)$ as the fundamental solution of the operator $\left(D^{\alpha}-\lambda\right)^{\circ j}$ :

$$
\mathcal{E}_{\alpha}^{\star j}(\lambda, t) \triangleq \sum_{k=0}^{\infty} C_{j-1+k}^{j-1} \lambda^{k} Y_{(j+k) \alpha}=t_{+}^{j \alpha-1} \sum_{k=0}^{\infty} C_{j-1+k}^{j-1} \frac{\left(\lambda t_{+}^{\alpha}\right)^{k}}{\Gamma((j+k) \alpha)}
$$

the Laplace transform of which is $\left(s^{\alpha}-\lambda\right)^{-j}$ for $\Re e(s)>a_{\lambda}$.

It follows that:

$$
\left(D^{\alpha}-\lambda\right)^{\circ j} \mathcal{E}_{\alpha}^{\star j}(\lambda, t)=\delta
$$


REMARK 2.12. With this extended definition $(j \geq 1)$, we have two notations for the same function in the case $j=1: \mathcal{E}_{\alpha}(\lambda, t)=\mathcal{E}_{\alpha}^{\star 1}(\lambda, t)$.

Remark 2.13. When $\alpha=1, \mathcal{E}_{1}(\lambda, t)=\exp (\lambda t) Y_{1}(t)$ is the causal exponential, the well-known fundamental solution of the operator $D^{1}-\lambda$.

REMARK 2.14. When $\alpha=1, \mathcal{E}_{1}^{\star j}(\lambda, t)=\exp (\lambda t) Y_{j}(t)$ is the causal polynomialexponential of degree $j-1$, the well-known fundamental solution of the operator $\left(D^{1}-\lambda\right)^{\circ . j}$.

\subsection{Stability Results for systems of Commensurate order}

2.2.1. Stability notions. Following [29, section 6.3], we recall the definition of external stability:

Definition 2.15. An input/ouput causal linear system defined by its impulse response $h$ is externally stable or bounded-input bounded-output (BIBO) iff:

$$
\forall u \in L^{\infty}\left(\mathbb{R}^{+}\right), \quad y=h \star u \in L^{\infty}\left(\mathbb{R}^{+}\right)
$$

which is satisfied when $h \in L^{1}\left(\mathbb{R}^{+}\right)$; in this case, we have:

$$
\|y\|_{\infty} \leq\|h\|_{1}\|u\|_{\infty}
$$

REMARK 2.16. A convolution kernel in $L^{1}\left(\mathbb{R}^{+}\right)$also provides other types of stabilities, namely:

- stability in the mean: $u \in L^{1}\left(\mathbb{R}^{+}\right) \Rightarrow y=h \star u \in L^{1}\left(\mathbb{R}^{+}\right)$, with:

$$
\|y\|_{1} \leq\|h\|_{1}\|u\|_{1}
$$

- stability in quadratic mean: $u \in L^{2}\left(\mathbb{R}^{+}\right) \Rightarrow y=h \star u \in L^{2}\left(\mathbb{R}^{+}\right)$, with:

$$
\|y\|_{2} \leq\|h\|_{1}\|u\|_{2}
$$

A sufficient condition for these stabilities is $h \in L^{1}\left(\mathbb{R}^{+}\right)$; but it must be noted that $h=K \delta+h_{1}$ with $h_{1} \in L^{1}\left(\mathbb{R}^{+}\right)$is a more general case, and $\|h\|_{1}$ must then be replaced by $|K|+|| h_{1} \|_{1}$ in (2.14), (2.15) and (2.16).

\subsubsection{Basic ELEMENTS.}

THEOREM 2.17. We have the following asymptotic equivalents for $\mathcal{E}_{\alpha}^{\star j}(\lambda, t)$ as $t$ reaches $+\infty$ :

- for $|\arg (\lambda)| \leq \alpha \pi / 2$,

$$
\left.\mathcal{E}_{\alpha}^{\star j}(\lambda, t) \sim \frac{1}{\alpha(j-1) !}\left\{\left(\frac{d}{d \sigma}\right)^{j-1} \sigma^{\frac{1}{\alpha}-1} \exp \left(\sigma^{\frac{1}{\alpha}} t\right)\right\}\right|_{\sigma=\lambda}
$$

it has the structure of a polynomial of degree $j-1$ in $t$, multiplied by $\exp \left(\lambda^{1 / \alpha} t\right)$. - for $|\arg (\lambda)|>\alpha \pi / 2$,

$$
\mathcal{E}_{\alpha}^{\star j}(\lambda, t) \sim \frac{\alpha}{\Gamma(1-\alpha)} j(-\lambda)^{-1-j} t^{-1-\alpha}
$$

which belongs to $L^{r}([1,+\infty[, \mathbb{R}), \forall r \geq 1$.

Proof. The proof of the theorem requires some analytic insight; we give a sketch of it in two steps:

- step 1: we compute the inverse Laplace transform of $\left(s^{\alpha}-\lambda\right)^{-j}$ with a cut along $\mathbb{R}^{-}$, in order to tackle the multiformity of the complex function under study (see [3, ch. III]). We are then left with a non zero residue term 
of polynomial-exponential type when $|\arg (\lambda)|<\alpha \pi$, and an integral term, namely:

$$
I_{\alpha}^{j}(\lambda, t)=\int_{0}^{+\infty} w_{\alpha, \lambda}^{j}(\beta) e^{-\beta t} d \beta
$$

the latter formula can be viewed as a continuous superposition of purely damped exponentials (it is therefore sometimes called the aperiodic multimode, see [25]);

- step 2: we then perform the asymptotic expansion near $t=+\infty$ of the integral term (2.19), which naturally proves to be strongly related to the fractional power series expansion at $\beta=0$ of the weight function or measure $w_{\alpha, \lambda}^{j}$ (see e.g. [4, chap. II, $\S 2.2$ ]; this is sometimes called the Watson lemma).

REMARK 2.18. in fact, the very diffusive nature ${ }^{2}$ of the non purely exponential part of the function $\mathcal{E}_{\alpha}^{\star j}(\lambda,$.$) is enlightened by formula (2.19).$

REMARK 2.19. the function $t \mapsto \mathcal{E}_{\alpha}^{\star j}(\lambda, t)$ is not continous at the origin $t=0$ in general; in fact, the following equivalent can be easily computed:

$$
\mathcal{E}_{\alpha}^{\star j}(\lambda, t) \sim Y_{j \alpha}(t)=\frac{t_{+}^{j \alpha-1}}{\Gamma(j \alpha)}
$$

which proves to be at least locally integrable at the origin.

Proposition 2.20. Basic elements $\mathcal{E}_{\alpha}^{\star j}(\lambda,$.$) define BIBO systems iff$

$$
|\arg \lambda|>\alpha \frac{\pi}{2}
$$

Proof. obvious from definition 2.15, theorem 2.17 and remark 2.19 .

2.2.3. Systems of commensurate order. Let us recall some now classical results on fractional differential equations (see [10, Appendix B] for a first reference and [12] later on):

Theorem 2.21. A transfer function $\mathcal{H}(s)=R\left(s^{\alpha}\right)$ for $\Re e(s)>a \geq 0$, where $R=Q / P$ stands for a rational function with $P$ and $Q$ two coprime polynomials, and $0<\alpha<1$ is the fractional order of derivatives, has the main property of:

$$
B I B O \text { stability } \Longleftrightarrow \quad|\arg \sigma|>\alpha \frac{\pi}{2}, \quad \forall \sigma \in \mathbb{C}, P(\sigma)=0
$$

In this latter case, the impulse response $h$ has the following asymptotics:

$$
h(t) \sim K t^{-1-\alpha} \quad \text { as } t \rightarrow+\infty
$$

REMARK 2.22. When $\sigma=0$ is a single root of $P, h(t) \sim K t^{-\alpha}$ as $t \rightarrow+\infty$, $h \notin L^{1}\left(\mathbb{R}^{+}\right)$and the system cannot be stable.

Proof. Let us introduce the partial fractions expansion of the rational function $R: R(\sigma)=\sum_{i=1}^{r} \sum_{j=1}^{\nu_{i}} \frac{r_{i j}}{\left(\sigma-\lambda_{i}\right)^{3}}$; then the impulse response $h$ can be computed as $h(t)=\sum_{i=1}^{r} \sum_{j=1}^{\nu_{i}} r_{i j} \mathcal{E}_{\alpha}^{\star j}\left(\lambda_{i}, t\right)$. Then, from theorem 2.17, we get the announced result.

Remark 2.23. For $\alpha=1$, this is the classical theorem of location of the poles of a BIBO system in the complex plane: no poles in the closed right-half plane.

${ }^{2}$ in the sense of $[23,9,21]$. 


\subsection{Stability Results For Systems of InCOMMENSURATE ORDER}

In this section, we will abandon the nice algebraic tools which proved useful in the preceding one, and we will strongly develop analytic reasonings: this will lead us to a much more comprehensive and much deeper understanding of the very nature of fractional $^{3}$ differential systems and definitely help us as far as the study of their stability is concerned.

\subsubsection{A genERAL RESULT.}

Theorem 2.24 (conjecture). Let $\mathcal{H}(s)=Q(s) / P(s)$ for $\Re e(s)>a \geq 0$ a transfer function, where $P(s)=\sum_{k=0}^{p} p_{k} s^{\alpha_{k}}$ with $\alpha_{k+1}>\alpha_{k} \geq 0$ and $Q(s)=\sum_{l=0}^{q} q_{l} s^{\beta_{l}}$ with $\beta_{l+1}>\beta_{l} \geq 0$ are no longer polynomials. The system has the main property of:

$$
B I B O \text { stability } \Longleftrightarrow \quad \exists M, \quad|\mathcal{H}(s)| \leq M \quad \forall s, \Re e(s) \geq 0
$$

Moreover, in the case where no simplification occurs between $P$ and $Q$ (that is $\forall s, \Re e(s) \geq 0, Q(s)=0 \Longrightarrow P(s) \neq 0)$, the stability property then reads:

$$
B I B O \text { stability } \Longleftrightarrow P(s) \neq 0 \quad \forall s, \Re e(s) \geq 0
$$

REMARK 2.25. Note that the condition $\Re e(s) \geq 0$ is very meaningful, because $\mathcal{H}(s)$ is uniquely determined in the closed right-half complex plane.

So far, this theorem is only a conjecture, and a rigorous proof can not be produced here. The only technicality to be proved is that such a transfer function $\mathcal{H}$ can not have an infinite number of singularities in the right-half complex plane, and that all these singularities are of finite order.

The second condition is more important in practise. The notion of simplification is not straightforward, let us give an example: $\mathcal{H}(s)=\frac{s^{\alpha}-1}{s-1}$ cannot be simplified in any way when $\alpha \notin \mathbb{Q}$; it seems that there is a singularity likely at $s=1$, but in fact $\mathcal{H}$ is continuous at $s=1$, and $\mathcal{H}(1)=\alpha$. Thus the system is stable when $\alpha \leq 1$.

REMARK 2.26. This theorem is not constructive in so far as it does not give any computable information, neither on the poles, nor on the asymptotics of the inverse Laplace transform $h(t)$ as $t$ reaches infinity.

The following theorem helps enlighten the structure of the system and can then be proposed in the following form:

THEOREM 2.27. For such a transfer function $\mathcal{H}(s)$, the corresponding impulse response can be decomposed into:

$$
h(t)=\sum_{i=1}^{r} \sum_{j=1}^{\nu_{i}} r_{i j} Y_{j}(t) e^{s_{i} t}+\int_{0}^{+\infty} \mu(x) e^{-x t} d x
$$

where $s_{i}$ are complex poles in $\mathbb{C} \backslash \mathbb{R}^{-}$and $\mu$ is a measure (which can be singular near $x=0$ ). If a singularity is to be found on the cut $\mathbb{R}^{-}$, then the integral term in (2.26) must be understood in the principal value sense of Cauchy (see [21]).

Moreover, the analytic form of the measure can be computed exactly:

$$
\mu(x)=\frac{1}{\pi} \frac{\sum_{k=0}^{p} \sum_{l=0}^{q} p_{k} q_{l} \sin \left(\left(\alpha_{k}-\beta_{l}\right) \pi\right) x^{\alpha_{k}+\beta_{l}}}{\sum_{k=0}^{p} p_{k}^{2} x^{2 \alpha_{k}}+\sum_{0 \leq k<l \leq p} 2 p_{k} p_{l} \cos \left(\left(\alpha_{k}-\alpha_{l}\right) \pi\right) x^{\alpha_{k}+\alpha_{l}}}
$$

\footnotetext{
${ }^{3}$ in fact, fractional should be reserved for systems of commensurate order, while non-integer would sound more appropriate for systems of incommensurate orders; but the word fractional has already been widely used in the literature, such as $[19,27]$.
} 
Sketch of the proof. See the two steps of the proof of theorem 2.17. The integral term comes from the cut along $\mathbb{R}^{-}$; it can be computed as follows:

$$
\mu(x)=\frac{1}{2 i \pi}\left[\mathcal{H}\left((-x)^{-}\right)-\mathcal{H}\left((-x)^{+}\right)\right]
$$

with $(-x)^{ \pm}=\exp ( \pm i \pi) x$ and $\left((-x)^{ \pm}\right)^{\alpha}=\exp ( \pm i \alpha \pi) x^{\alpha}$; some tedious computations then follow and lead to equation $(2.27)$.

REMARK 2.28 (To what extent is decomposition (2.26) structural?). The transfer function $\mathcal{H}$ is uniquely determined in a right-half plane $\Re e(s)>a \geq$ 0 , where it is analytic. The impulse response $h$ is computed using the inverse Laplace transform, namely $h(t)=\frac{1}{2 i \pi} \int_{c-i \infty}^{c+i \infty} \mathcal{H}(s) e^{s t} d s$, where $c>a$. Now, in order to use classical residue calculus, a closed path must be defined, in which $\mathcal{H}$ is meromorphic: a cut must therefore be perfomed between the two branching points $s=0$ and " $|s|=+\infty$, provided $\Re e(s)<0$ "; the choice of the path of integration is free, and the result $h$ does not depend upon the computation method. A commonly used choice is a cut along $\mathbb{R}^{-}$: it is the most reasonable one, because it preserves hermitian properties for the roots, and because it clearly separates between classical dynamics (linear differential systems of integer order) on the one hand and infinite-dimensional dynamics (namely diffusions) on the other hand; this intrinsic decomposition can be called structural. Now it seems obvious that changing the cut will strongly affect the poles in the open left-half complex plane; also the measure $\mu$ will change: therefore we are urged at defining what is structural and what is not.

- the poles $s_{i}$ with $\Re e\left(s_{i}\right) \geq 0$, their order and residues are structural elements. The poles $s_{i}$ with $\Re e\left(s_{i}\right)<0$ are also structural if and only if it has been made clear that a cut along $\mathbb{R}^{-}$has been used; otherwise they highly depend on the choice of the cut to perform the computation (straightforward exercise left to the reader), and thus can not be called structural.

- concerning the integral term, its asymptotic expansion is structural, and it is highly unique, given by the expansion at the origin $x=0$ of the measure $\mu$ only. (Note that it does not depend on the cut that is chosen to perform the computation, thus proving to be a fully structural component of the decomposition). More precisely, this integral term can be decomposed into some pure integrators of fractional orders $Y_{\nu_{n}}(t)$ with $\nu_{n}>0$ and a regular term in $L^{1}\left(\mathbb{R}^{+}\right)$, which behaves like $\mu_{1} t^{-1-\gamma_{1}}$ asymptotically.

Now in order to be complete, we need to give the asymptotics of the regular part of the integral term, which appears in the right-hand side of (2.26), we recall the Watson lemma:

$$
\mu(x)=\sum_{m=1}^{M} \mu_{m} \frac{x^{\gamma_{m}}}{\Gamma\left(1+\gamma_{m}\right)}+o\left(x^{\gamma_{M}}\right) \Longrightarrow I(t)=\sum_{m=1}^{M} \mu_{m} \frac{1}{t^{1+\gamma_{m}}}+o\left(t^{-1-\gamma_{M}}\right)
$$

REMARK 2.29. a very particular case occurs when fractional differential systems of commensurate order are dealt with, for $\alpha_{k}=k \alpha$ and also $\beta_{l}=l \alpha$; then it is clear that only $\gamma_{m}=m \alpha$ are to be found in (2.29), thus implying an asymptotic expansion in $\sum_{m} \mu_{m} t^{-1-m \alpha}$.

2.3.2. USING DIFFUSIVE BALANCED REALISATIONS. Let us now introduce the socalled diffusive balanced realisations of fractional integrals and derivatives. These infinite-dimensional formulations will help us prove some crucial positivity properties which will be of major help in the studies of stability. 
First consider the infinite-dimensional dynamical system with input $u$, ouput $y$ and state $\varphi(0<\beta<1)$ :

$$
\begin{aligned}
\partial_{t} \varphi(\xi, t) & =-\xi \varphi(\xi, t)+\sqrt{\mu_{\beta}(\xi)} u(t) ; \quad \varphi(\xi, 0)=0, \quad \forall \xi \in \mathbb{R}^{+} \\
y(t) & =\int_{0}^{+\infty} \sqrt{\mu_{\beta}(\xi)} \varphi(\xi, t) d \xi
\end{aligned}
$$

with $\mu_{\beta}(\xi)=\frac{\sin (\beta \pi)}{\pi} \xi^{-\beta}$, a positive measure on $\mathbb{R}^{+}$.

THEOREM 2.30. The input-output relation for system (2.30)-(2.31) is $y(t)=I^{\beta} u(t)$. Moreover, we have the positivity property:

$$
\forall T>0, \quad<y, u>_{T}=\int_{0}^{T} y(t) u(t) d t \geq 0
$$

Proof. we refer to $[23,22]$ and references therein. Note that functional spaces must be specified for these infinite-dimensional dynamical systems to make sense; in particular, a classical $V \subset H \subset V^{\prime}$ framework is needed.

Property $(2.32)$ can be found formally by computation:

$$
\frac{1}{2} \int_{0}^{+\infty} \varphi^{2}(\xi, T) d \xi+\int_{0}^{T} \int_{0}^{+\infty} \xi \varphi^{2}(\xi, t) d \xi d t=<y, u>_{T}
$$

it then makes full sense in the appropriate functional spaces: $u \in L^{2}(0, T ; \mathbb{R})$ implies $y \in L^{2}(0, T ; \mathbb{R})$, and the scalar product is well defined.

Now consider the infinite-dimensional dynamical system with input $u$, ouput $z$ and state $\psi(0<\alpha<1)$ :

$$
\begin{aligned}
\partial_{t} \psi(\xi, t) & =-\xi \psi(\xi, t)+\sqrt{\mu_{1-\alpha}(\xi)} u(t) ; \quad \psi(\xi, 0)=0, \quad \forall \xi \in \mathbb{R}^{+}(2 \\
z(t) & =\int_{0}^{+\infty} \sqrt{\mu_{1-\alpha}(\xi)}\left(-\xi \psi(\xi, t)+\sqrt{\mu_{1-\alpha}(\xi)} u(t)\right) d \xi
\end{aligned}
$$

THEOREM 2.31. The input-output relation for system (2.34)-(2.35) is $z(t)=D^{\alpha} u(t)$. Moreover, we have the positivity property:

$$
\forall T>0, \quad<z, u>_{T}=\int_{0}^{T} z(t) u(t) d t \geq 0
$$

Proof. formally, $z=D^{\alpha} u=D^{1} \circ I^{1-\alpha} u$; then use $\varphi$ with $\beta=1-\alpha$. We refer to $[23,22]$ and references therein. Once again, functional spaces must be specified for these infinite-dimensional dynamical systems to make sense; in particular, a classical $V \subset H \subset V^{\prime}$ framework is needed.

Property (2.36) can be found formally by specific computation:

$$
\frac{1}{2} \int_{0}^{+\infty} \xi \psi^{2}(\xi, T) d \xi+\int_{0}^{T} \int_{0}^{+\infty}\left(\partial_{t} \psi\right)^{2}(\xi, t) d \xi d t=\left\langle z, u>_{T}\right.
$$

it then makes full sense in the appropriate functional spaces: $u \in H^{1}(0, T ; \mathbb{R})$ implies $z \in L^{2}(0, T ; \mathbb{R})$, and the scalar product is well defined.

REMARK 2.32. In (2.35), the two parts can not be evaluated separately, otherwise the integrals would both diverge. This is very well understood in the appropriate functional framework.

REMARK 2.33. Diffusive realisations of fractional integrals $I^{\beta}$ and derivatives $D^{\alpha}$, and other pseudo-differential operators, are very important both from theoretical and numerical viewpoints: 
- from a theoretical aspect, these formulations help understand the very nature of fractional integrals and derivatives (as a particular case of long-memory operators), they also provide natural and straightforward proofs for properties which would otherwise not be so obvious;

- from a practical aspect, these formulations help define stable numerical schemes for the approximation of the solution of such systems.

In section 2.4, we will use the positivity properties (2.32) and (2.36) in order to find sufficient stability conditions for fractional differential systems of commensurate or incommensurate orders. Moreover diffusive realisations of fractional integrals or derivatives through a state of infinite dimension $\varphi$ or $\psi$ help define a natural state space, together with appropriate energy functionals, which are both most useful for stability considerations.

\subsection{Some case STUdies}

We propose to study the following very simple examples in order to show the combined interest of both algebraic and analytic approaches.

2.4.1. A first order system with Fractional Damping. Consider the following first order stable $(\tau>0)$ system perturbed $(\varepsilon \neq 0)$ by some fractional damping of order $\alpha \in] 0,1[$ :

$$
\tau \dot{x}+\varepsilon D^{\alpha} x+x=u
$$

Using energy methods and diffusions, we can prove that the system is stable $\forall \varepsilon>0$. Let us introduce the global energy functional of the augmented system:

$$
E(t)=\frac{\tau}{2} x^{2}(t)+\varepsilon E_{\psi}(t)
$$

where the energy of the diffusive variable $\psi$ is $E_{\psi}(t)=\frac{1}{2} \int_{0}^{+\infty} \xi \psi^{2}(\xi, t) d \xi>0$, and compute formally:

$$
\dot{E}(t)=\tau x \dot{x}+\varepsilon \dot{E}_{\psi}(t)=x u-x^{2}-\varepsilon \int_{0}^{t} \int_{0}^{+\infty}\left(\partial_{t} \psi\right)^{2}(\xi, \tau) d \xi d \tau
$$

Then as soon as the input $u$ has stopped, the global energy starts decreasing; finally, LaSalle principle will help prove that the augmented state $(x, \psi)$ of the global system goes to $(0,0)$ as $t \rightarrow+\infty$.

But this sufficient condition is not necessary at all: when $\alpha=1 / 2$ for example, $\sigma^{2}-2 \rho \cos (\theta) \sigma+\rho^{2}$ has stable poles for $|\theta|>\pi / 4$ (which is the most accurate stability result); thus when $\pi / 4<|\theta|<\pi / 2$, the system is still stable whereas $\varepsilon=-2 \tau \rho \cos (\theta)<0$.

REMARK 2.34. Using energy methods, it is internal stability that is in fact examined; and contrarily to the finite-dimensional case, internal stability need not imply external stability. But in this particular case, it can be proved that $\forall s, \Re e(s) \geq 0$, $\Re e\left(\tau s+\varepsilon s^{\alpha}+1\right)=\tau \rho \cos (\theta)+\varepsilon \rho^{\alpha} \cos (\alpha \theta)+1 \neq 0$ when $\varepsilon>0$; then, applying theorem 2.24, we find that the system is also externally stable.

2.4.2. A second order system With fractional dampings. Consider the following second order stable $(\varepsilon>0)$ system perturbed $(p q \neq 0)$ by some fractional dampings of order $1+\alpha \in] 1,2[$ and $1-\beta \in] 0,1[$ :

$$
\ddot{x}+p D^{\alpha} \dot{x}+\varepsilon \dot{x}+q I^{\beta} \dot{x}+\omega^{2} x=u
$$

Using energy methods and diffusions, we can prove that the system is stable $\forall p>0$ and $\forall q>0$. Let us introduce the global energy functional of the augmented 
system:

$$
E(t)=\frac{1}{2} \dot{x}^{2}(t)+\frac{\omega^{2}}{2} x^{2}(t)+p E_{\psi}(t)+q E_{\varphi}(t)
$$

where the energy of the diffusive variable $\varphi$ is $E_{\varphi}(t)=\frac{1}{2} \int_{0}^{+\infty} \varphi^{2}(\xi, t) d \xi>0$, and the energy of the diffusive variable $\psi$ is $E_{\psi}(t)=\frac{1}{2} \int_{0}^{+\infty} \xi \psi^{2}(\xi, t) d \xi>0$. Let us compute, at least formally:

$$
\begin{aligned}
\dot{E}(t)= & \dot{x} \ddot{x}+\omega^{2} x \dot{x}+p \dot{E}_{\psi}(t)+q \dot{E}_{\varphi}(t) \\
= & \dot{x} u-\varepsilon \dot{x}^{2}-p \int_{0}^{t} \int_{0}^{+\infty}\left(\partial_{t} \psi\right)^{2}(\xi, \tau) d \xi d \tau \\
& -q \int_{0}^{t} \int_{0}^{+\infty} \xi \varphi^{2}(\xi, \tau) d \xi d \tau
\end{aligned}
$$

Then as soon as the input $u$ has stopped, the global energy starts decreasing; finally, LaSalle principle will help prove that the augmented state $(x, \dot{x}, \psi, \varphi)$ of the global system goes to $(0,0,0,0)$ as $t \rightarrow+\infty$.

But this sufficient condition is not necessary at all: when $\alpha=1 / 2$ for example, $\sigma^{4}+p \sigma^{3}+\varepsilon \sigma^{2}+q \sigma+\omega^{2}$ has stable poles iff $\left|\arg \sigma_{k}\right|>\pi / 4$; this can lead very easily to $p=-\sum_{k} \sigma_{k}<0$ and $q=-\sum_{i, j, k} \sigma_{i} \sigma_{j} \sigma_{k}<0$, but still $\varepsilon=\sum_{k, l} \sigma_{k} \sigma_{l}>0$ and $\omega^{2}=\prod_{k} \sigma_{k}>0$ (numerical examples are left to the reader).

REMARK 2.35. Using energy methods, it is internal stability that is in fact examined; and contrarily to the finite-dimensional case, internal stability need not imply external stability. In this particular case, we can only conjecture that the system is also externally stable.

2.4.3. Conclusion. The two preceding examples show how complementary the algebro-analytic and purely analytic methods can prove to be. Such a coherence between the two approaches has already been noticed and used (see e.g. [14] where the energy decay was obtained through diffusive realisations, and asymptotics were computed using a so-called fractional modal decomposition).

\section{Generalized fractional differential systems}

\subsection{Generalized Basic elements}

Considering $s_{0} \in \mathbb{C}$ instead of 0 as branching point leads us to define the following basic element in the Laplace domain: $\left(\left(s-s_{0}\right)^{\alpha}-\lambda\right)^{-1}$ for $\Re e(s)>a_{\lambda, s_{0}}$ (a cut being performed along the half-line $\left.\left.]-\infty+i \Im m\left(s_{0}\right), s_{0}\right]\right)$, which proves to be the fundamental solution of the generalized fractional differential operator $D_{s_{0}}^{\alpha}-\lambda$ in the functional space of causal tempered distributions $\mathcal{S}_{+}^{\prime}$, with:

$$
D_{s_{0}}^{\alpha} T \triangleq \exp \left(s_{0} t\right) D_{0}^{\alpha}\left(\exp \left(-s_{0} t\right) T\right)
$$

We can state the following stability results for these basic elements:

Proposition 3.1. Let us denote $\mathcal{E}_{\alpha, s_{0}}(\lambda, t)=\mathcal{L}^{-1}\left[\left(\left(s-s_{0}\right)^{\alpha}-\lambda\right)^{-1}\right]$, then:

- when $\Re e\left(s_{0}\right)>0$, these generalized basic elements are all unstable, whatever the location of $\lambda$ in the complex plane,

- when $s_{0}=i \omega_{0}$, the region of stability (namely $|\arg \sigma|>\alpha \pi / 2$ ) is fully preserved, but the stable dynamics now behave like:

$$
K_{\lambda} t^{-1-\alpha} e^{i \omega_{0} t} \quad \text { as } t \rightarrow+\infty
$$


- when $\Re e\left(s_{0}\right)<0$, the region of instability in $\mathbb{C}$ is shrunk (namely, the interior of the limiting curve

$$
|\sigma|^{1 / \alpha} \cos ((\arg \sigma) / \alpha)=-\left|s_{0}\right| \cos \left(\arg s_{0}\right)
$$

in the formerly unstable sector $|\arg \sigma| \leq \alpha \pi / 2)$; the stable dynamics then behave like:

$$
K_{\lambda} t^{-1-\alpha} e^{s_{0} t} \quad \text { as } t \rightarrow+\infty
$$

Proof. The proof is straightforward, once it has been made clear that $\mathcal{E}_{\alpha_{,} s_{0}}(\lambda, t)=$ $\exp \left(s_{0} t\right) \mathcal{E}_{\alpha, 0}(\lambda, t)$.

REMARK 3.2. Note that when $\lambda=0$, the asymptotics is in $K_{0} t^{-\alpha} e^{s_{0} t}$; in this case only, the branching point $s_{0}$ is a singularity in the neighborhood of which the function goes to infinity (like a pole for a meromorphic function), in the sense that:

$$
\lim _{s \rightarrow s_{0}}\left|\left(s-s_{0}\right)^{-\alpha}\right|=+\infty
$$

REMARK 3.3. From a modelling point of view, the case $\Re e\left(s_{0}\right)=0$ is the most interesting, because a new variety of anomalous decays are being captured.

\subsection{Generalized systems}

From the careful asymptotic analysis of the previously defined generalized basic elements, we can now define systems in a straightforward way.

Definition 3.4. A generalized fractional differential (single-input single-output) system of commensurate order $\alpha$ is defined by:

$$
P\left(D_{s_{0}}^{\alpha}\right) y(t)=Q\left(D_{s_{0}}^{\alpha}\right) u(t)
$$

with input $u$ and output $y$.

It is now clear that such a system is BIBO-stable iff all the poles of the rational function $R$ in the $\sigma$-plane lie in the open stability region defined in $\S 3.1$. In this latter case, the impulse response of the generalized system behaves like:

$$
K t^{-1-\alpha} e^{s_{0} t} \quad \text { as } t \rightarrow+\infty
$$

\subsection{Other generalized SyStems AND APPLiCATIONS}

We examined the possibility of having a pole that is disconnected from the branching point, and then we put these elementary systems in cascade, all of them sharing the same $\alpha$ and the same $s_{0}$; in fact, this amounts to considering rational functions $R$ in the variable $\sigma=\left(s-s_{0}\right)^{\alpha}$, hence providing a much better structured setting which allows for a fully algebraic treatment of such systems.

But other generalized systems can be defined as well: the $\alpha$ and $s_{0}$ can vary from one system to the other; we still get nice properties if only series of such systems are considered. Otherwise the analysis of the whole system is not straightforward: in [30] for example, cascade of elements with different $\alpha$ and $s_{0}$ but same $\lambda=0$ are considered.

Let us examine an interesting example of application: simulation, convolution and asymptotic expansion of some intrinsically pseudo-differential function, namely a causal Bessel function of the first kind and of order $0, h=J_{0}$, the Laplace transform of which is:

$$
\mathcal{H}(s)=\frac{1}{\sqrt{s^{2}+1}} \forall s, \Re e(s)>0
$$

Finding the first term of the asymptotic expansion is straightforward thanks to the results of the preceding section. We have two branching points $s_{0}=i$ and $s_{1}=-i$ : two cuts can be performed, one along the half-line $]-\infty+i$, $i$ ] and 
another one along the half-line $]-\infty-i,-i]$. In this doubly cut complex plane, we have the identity $\sqrt{s^{2}+1}=\sqrt{s-i} \sqrt{s+i}$, where $\sqrt{s}$ is uniquely determined on $\mathbb{C} \backslash \mathbb{R}$ - by analytic continuation. Then, in the vicinity of $s_{0}=i, \mathcal{H}(s) \sim \frac{1}{\sqrt{2 i}} \frac{1}{\sqrt{s-i}}=$ $\frac{1}{\sqrt{2}} \exp (-i \pi / 4) \frac{1}{\sqrt{s-i}}$, which possess the asymptotics $\frac{1}{\sqrt{2} \Gamma(1 / 2)} t^{-1 / 2} \exp (i(t-\pi / 4))$. In the vicinity of $s_{1}=s_{0}^{*}$, we get the conjugate estimate, thus leading to the wellknown asymptotic expansion for $J_{0}(t)$ :

$$
h(t) \sim \sqrt{\frac{2}{\pi t}} \cos \left(t-\frac{\pi}{4}\right)
$$

A purely diffusive formulation of $h$ can also be given, namely:

$$
\begin{aligned}
h(t)= & -\frac{1}{\pi} e^{i t} \int_{0}^{+\infty} \frac{1}{\sqrt{x} \sqrt{-x+2 i}} e^{-x t} d x \\
& -\frac{1}{\pi} e^{-i t} \int_{0}^{+\infty} \frac{1}{\sqrt{x} \sqrt{-x-2 i}} e^{-x t} d x
\end{aligned}
$$

from which diffusive realisation directly follows, which reveals helpful for computation (numerical simulation) of $h$ itself, or of convolutions by $h$. Formulation (3.9) also enables to compute higher order asymptotics if needed.

\section{CONCLUSION}

In this paper, external stability of linear fractional differential systems has been extensively studied: the case of commensurate order happens to be a very particular case of the case of incommensurate orders; the asymptotics of the impulse response can be computed in the most general case, and are somehow revealed by diffusive realisations. Some striking, though elementary, examples are dealt with, which show the combined interest of both analytic and algebraic approaches. Moreover, extending these techniques to generalized fractional differential systems proves very much useful, with special application in simulation, convolution and asymptotic expansion of some special functions, such as Bessel functions, which possess an intrinsic pseudo-differential nature.

In the very near future, these considerations will be extended to discrete-time systems, and discrete-time diffusive realisations will be introduced in order to deal with stability problems of fractional difference systems, and also to reveal the very diffusive nature of such systems (which might have been overlooked so far in the literature).

\section{REFERENCES}

[1] H. Bateman. Higher transcendental functions, volume 3, chapter XVIII, pages 206-212. McGraw Hill, New York, 1954.

[2] P. L. Butzer. Singular integral equations of Volterra type and the finite part of divergent integrals. Arch. Rat. Mech. and Anal., 3:194-205, 1959.

[3] B. Chabat. Introduction à l'analyse complexe, volume 1. Mir, 1985. (transl. from Russian, 1990).

[4] A. Erdélyi. Asymptotic expansions. Dover, 1956.

[5] Calcul opérationnel et fonctions généralisées. Dunod, 1971.

[6] M. Fliess and R. Hotzel. Sur les systèmes linéaires à dérivation non entière. C. R. Acad. Sci. Paris Sér. IIb, 324:99-105, 1997.

[7] R. Gorenflo and F. Mainardi. Fractional oscillations and Mittag-Leffler functions. Technical Report preprint A-14/96, Freie Universität Berlin, 1996.

[8] I. M. Guelfand and G. E. Chilov. Les distributions, tome 1, volume 8 of Monographies Universitaires de Mathématiques. Éditions Dunod, 1972. (transl. from Russian).

[9] D. Heleschewitz and D. Matignon. Diffusive realisations of fractional integrodifferential operators: structural analysis under approximation. In Conference IFAC System, Structure and Control, volume 2, pages 243-248, Nantes, France, July 1998. IFAC. 
[10] D. Matignon. Représentations en variables d'état de modèles de guides d'ondes avec dérivation fractionnaire. Thèse de Doctorat, Univ. Paris XI, Novembre 1994.

[11] _ Fractional modal decomposition of a boundary-controlled-and-observed infinitedimensional linear system. Saint Louis, Missouri, June 1996. mTns. 5 pages, presented at the Mathematical Theory of Networks and Systems symposium.

[12] Stability results for fractional differential equations with applications to control processing. In Computational Engineering in Systems Applications, volume 2, pages 963-968, Lille, France, July 1996. IMACS, IEEE-SMC.

[13] Generalized fractional differential and difference equations: stability properties and modelling issues. In Mathematical Theory of Networks and Systems symposium, Padova, Italy, July 1998. mTNs. 4 pages, accepted for publication.

[14] D. Matignon, J. Audounet, and G. Montseny. Energy decay for wave equations with damping of fractional order. In Fourth international conference on mathematical and numerical aspects of wave propagation phenomena, pages 638-640, Golden, Colorado, June 1998. INRIA, SIAM.

[15] D. Matignon and B. d'Andréa-Novel. Spectral and time-domain consequences of an integrodifferential perturbation of the wave PDE. In Third international conference on mathematical and numerical aspects of wave propagation phenomena, pages 769-771, Mandelieu, France, April 1995. INRIA, SIAM.

[16] Some results on controllability and observability of finite-dimensional fractional differential systems. In Computational Engineering in Systems Applications, volume 2, pages 952-956, Lille, France, July 1996. IMACS, IEEE-SMC.

[17] . Observer-based controllers for fractional differential systems. In Conference on Decision and Control, pages 4967-4972, San Diego, California, December 1997. IEEE-CSS, SIAM.

[18] B. Mbodje and G. Montseny. Boundary fractional derivative control of the wave equation. IEEE Trans. Aut. Cont., 40(2):378-382, Feb. 1995.

[19] K. S. Miller and B. Ross. An introduction to the fractional calculus and fractional differential equations. John Wiley \& Sons, 1993.

[20] G. Mittag-Leffler. Sur la représentation analytique d'une branche uniforme d'une fonction monogène. Acta Math., 29:101-168, Sept. 1904.

[21] G. Montseny. Diffusive representation of pseudo-differential time-operators. ESA IM:Proceedings, 5:159-175, December 1998. URL: http://ннн .emath .fr/proc/vol.5/.

[22] G. Montseny, J. Audounet, and D. Matignon. Fractional integrodifferential boundary control of the Euler-Bernoulli beam. In Conference on Decision and Control, pages 4973-4978, San Diego, California, December 1997. IEEE-CSS, SIAM.

[23] G. Montseny, J. Audounet, and B. Mbodje. Optimal models of fractional integrators and application to systems with fading memory. In Conference IEEE Systems, Man and Cybernetics, pages 65-70, Le Touquet, France, October 1993. IEEE-SMC.

[24] K. B. Oldham and J. Spanier. The Fractional Calculus. Academic Press, New York and London, 1974

[25] A. Oustaloup. Systèmes asservis linéaires d'ordre fractionnaire. Série Automatique. Masson, 1983.

[26] I. Podlubny. The Laplace transform method for linear differential equations of the fractional order. Technical Report UEF-02-94, Inst. Exp. Phys., Slovak Acad. Sci., Kosice, 1994.

[27] S. G. Samko, A. A. Kilbas, and O. I. Marichev. Fractional integrals and derivatives: theory and applications. Gordon \& Breach, 1987. (transl. from Russian, 1993).

[28] L. Schwartz. Méthodes mathématiques pour les sciences physiques. Coll. Enseignement des Sciences. Hermann, 1965.

[29] E. D. Sontag. Mathematical Control Theory. Deterministic Finite Dimensional Systems, volume 6 of Texts in Applied Mathematics. Springer Verlag, 1990.

[30] M.-C. Viano, C. Deniau, and G. Oppenheim. Continuous time fractional ARma processes. Stat. Prob. Letters, 21:323-336, 1994. 